\title{
Analysis of Wireless Power Transfer Characteristics for Multiple Receivers by Time Sharing Technique
}

\author{
Jongmin Park $\cdot$ Sangwook Nam
}

\begin{abstract}
A multiple charging method for a wireless power transfer system (WPTS) in the near-field region is proposed. We analyzed the frequency characteristics of multiple receivers in the near-field region. The results suggested that the time division WPTS can achieve efficient and equal power transmission at multiple receivers. We conclude that this system has an advantage for charging multiple receivers.
\end{abstract}

Key words: Multiple Charging, Time Sharing, Wireless Power Transfer.

\section{Introduction}

WPT has been studied for many years [1]. Recently, WPT techniques using resonant coupling has been developed intensively [2] [5]. The efficiency of the WPT system using near-field coupling is very high at a close range. Previous work [6] [9] suggested that the frequency tracking method could be used for adaptive matching. This method controls the frequency of the WPTS source based on the coupling between the antennas. However, the method has various limitations, including the case of multiple receivers charging. To address this issue, we investigated the characteristics of multiple receivers and propose that the time division WPTS resolves this problem.

\section{II . Frequency Characteristics of Multiple Receivers}

The adaptive matching method for WPT using frequency tracking has been studied by many groups. However, the method underlying multiple receivers charging remains unclear. The frequency characteristic of one receiver is greatly different from that of multiple receivers. Fig. 1 shows the antennas structure and the positions of the multiple receivers. FEKO was used as the simulator. The set-up of the center-fed helical dipole antenna is shown in Fig. 1. The diameter of the wire was $1 \mathrm{~mm}$, and the height and diameter of the antenna were $6 \mathrm{~cm}$ and $7 \mathrm{~cm}$, respectively. The resonant frequency was $13.56 \mathrm{MHz}$. The distance between the transmitter and the first receiver was fixed at $15 \mathrm{~cm}$. The angle between the transmitter and the receivers was set at $60^{\circ}$.
The port impedance at the antennas was $50 \Omega$. Fig. 2 shows the power transfer efficiency (PTE) based on the frequency when the distance between the transmitter and the second receiver was $20 \mathrm{~cm}$. The PTE is defined as:

$$
\mathrm{PTE}=\frac{\mathrm{P}_{L}}{\mathrm{P}_{a v s}}
$$

where $\mathrm{P}_{L}$ is the power delivered to the load, $\mathrm{P}_{a v s}$ is the power available from the source. The total PTE and the difference between the individual power transfer efficiencies are shown in Fig. 2. Generally, the goal of any power transfer system for multiple receivers is to obtain efficient and equal charging characteristics. At the frequency where the total PTE was efficient, the difference in the PTE of the first and the second receiver was quite high. Therefore, only one of the receivers was well charged, and the others were hardly charged at all. Although the PTE of the first receiver is high when the second receiver is nonexistent, the almost power from the source is transmitted to the second receiver when the coupling between the source and the second receiver is stronger

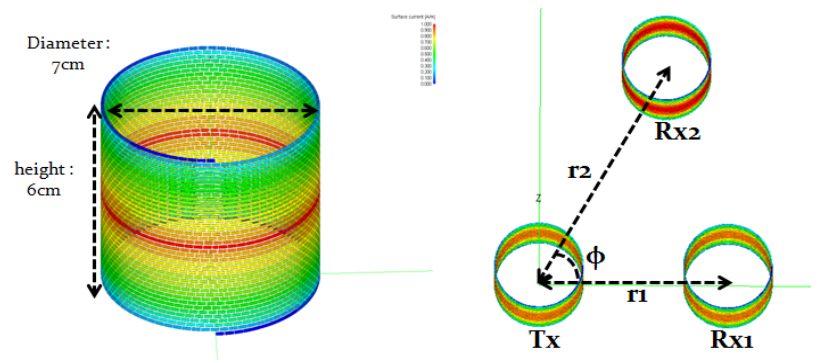

Fig. 1. Structure of the multiple receivers charging system.

Manuscript received August 30, 2011 ; revised September 16, 2011. (ID No. 20110830-3J)

The School of Electrical Engineering and INMC, Seoul National University, Seoul, Korea.

Corresponding Author : Jongmin Park (e-mail : city814@ael.snu.ac.kr) 
than the coupling between the source and first receiver. The equal charging characteristics can be realized only under the condition that the couplings are same. Such a situation is not advisable.

\section{Characteristics of the Time Division WPTS}

The analysis at the previous charter highlighted the difficulties surrounding the charging of multiple receivers. To resolve this problem, we propose the time division WPTS. Generally, the WPTS operates at a very low frequency, with the result that the size of the antennas in the system is very small. Therefore, we can assume that an antenna in a WPTS is a quasi-canonical minimum scattering (CMS) antenna. A CMS antenna is defined as one that becomes invisible when the antenna port is open-circuited [10]. Fig. 3 shows the proposed circuit of the receiver at the time division WPTS. The switch is added at the port of the receivers. When the

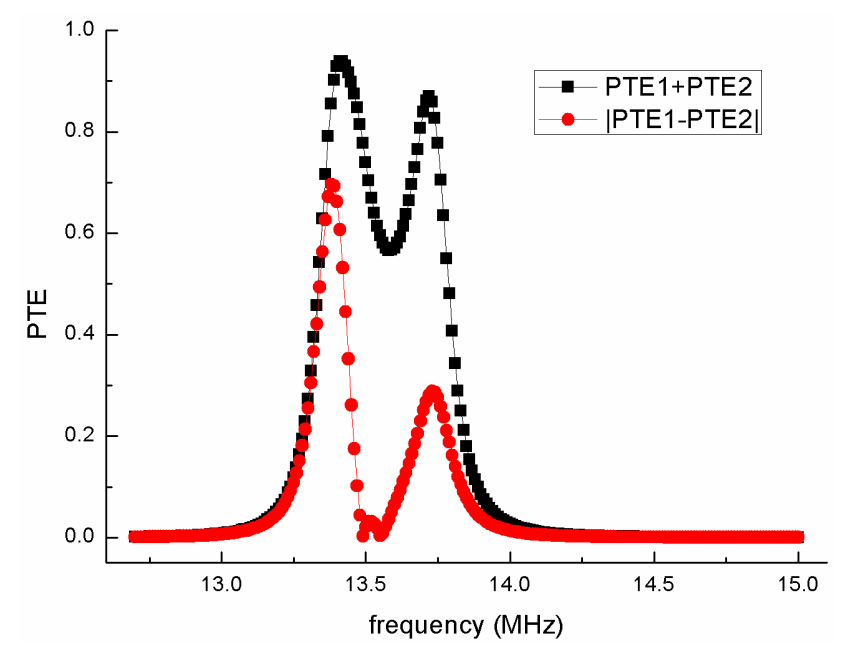

Fig. 2. Total power transfer efficiency and the difference between the individual power transfer efficiencies $\left(r_{1}=15 \mathrm{~cm}, r_{2}=20 \mathrm{~cm}\right)$.

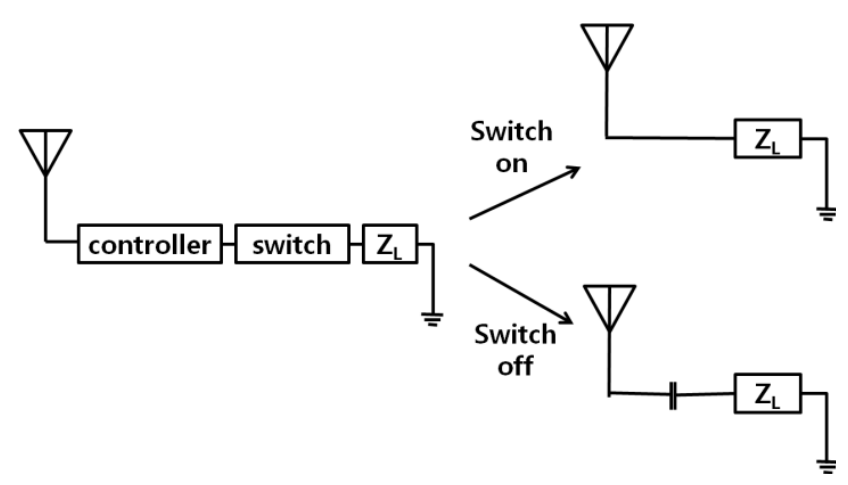

Fig. 3. Equivalent circuit of the receiver according to the switch state. switch is off, the load impedance resembles a small capacitor. Therefore, the receiver port is almost opencircuited condition and then the receiver is invisible. As a result, the transmitter can transmit power at the other receiver efficiently.

The controller at the receiver controls the switch, which is connected during the allotted time. The PTE of each receiver according to the switch state is shown in Figs. 4 and 5. When the switch of the second receiver was off, the PTE was almost same as that of the case of the without the second receiver as shown in Fig. 4. Similarly, when the switch of the first receiver was off, the power was efficiently transmitted to the second receiver as shown in Fig. 5.

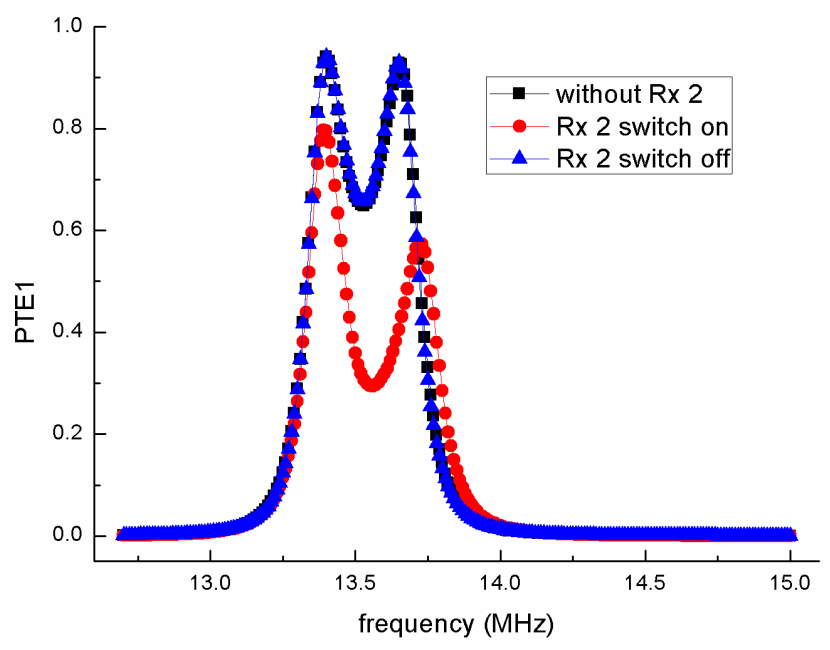

Fig. 4. Power transfer efficiency of the first receiver according to the switch state of the second receiver $\left(r_{1}\right.$ $=15 \mathrm{~cm}, r_{2}=20 \mathrm{~cm}$ ).

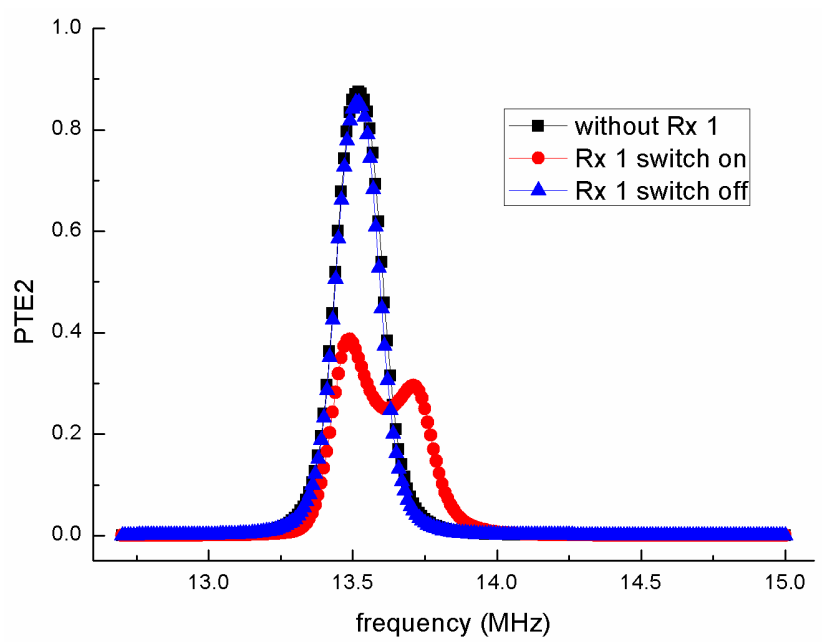

Fig. 5. Power transfer efficiency of the second receiver according to the switch state of the first receiver $\left(r_{1}=15 \mathrm{~cm}, r_{2}=20 \mathrm{~cm}\right)$. 


\section{Conclusion}

In this paper, the characteristics of the WPTS to charge the multiple receiver is analyzed. We conclude that in terms of multiple receiver' charging, the proposed time division WPTS can transmit power efficiently and equally.

This research was supported by the KCC (Korea Communications Commission), Korea, under the R\&D program supervised by the KCA (Korea Communications Agency) (KCA-2011- (11911-01110)).

\section{References}

[1] N. Tesla, "Apparatus for transmitting electrical energy," U.S. Patent 1119 732, Dec. 1914.

[2] A. Kurs, A. Karalis, R. Moffatt, J. D. Joannopoulos, P. Fisher, and M. Soljacic, "Wireless power transfer via strongly coupled magnetic resonances," Sciencexpress, Jun. 2007.

[3] J. Lee, S. Nam, "Fundamental aspects of near-field coupling antennas for wireless power transfer," IEEE Trans. Antennas and Propag., vol. 58, no. 11, pp. 3442-3449, Nov. 2010.

[4] G. Kim, Y. Jung, and B. Lee, "Wireless power transmission between two metamaterial-inspired loops at $300 \mathrm{MHz}$," Journal of Korean Institute Electronic Engineering and Science, vol. 10, no. 4, Dec. 2010.

[5] I. Awai, T. Ishida, "Design of resonator-coupled wireless power transfer system by use of BPF Theory," Journal of Korean Institute Electronic Engineering and Science, vol. 10, no. 4, Dec. 2010.

[6] Y. Kim, H. Ling, "Investigation of coupled mode behaviour of electrically small meander antennas," Electron. Lett., vol. 43, no. 23, Nov. 2007.

[7] W. Fu, B. Zhang, and D. Qiu, "Study on frequency-tracking wireless power transfer system by resonant coupling," Power Electronics and Motion Control Conf., IPEMC. IEEE 6th International 2009, pp. 2658-2663, 2009.

[8] A. P. Sample, D. A. Meyer, and J. R. Smith, "Analysis, experimental results, and range adaptation of magnetically coupled resonators for wireless power transfer," IEEE Trans. Industrial Electronics, vol. 58, pp. 544-554, Feb. 2011.

[9] J. Park, Y. Tak, Y. Kim, Y. Kim, and S. Nam, "Investigation of adaptive matching methods for near field wireless power transfer," IEEE Trans. Antennas and Propag., vol. 59, no. 5, May 2011.

[10] W. K. Kahn, H. Kurss, "Minimum-scattering antennas," IEEE Trans. Antennas and Propag., vol. 13, no. 5, pp. 671-675, Sep. 1965. 